\title{
Controle da Anemia Ferropriva em Pré-Escolares por Meio da Fortificação de Alimento Com Concentrado de Hemoglobina Bovina (Estudo Preliminar)
}

\author{
Iron Deficiency Anemia Control in Pre-School Children by Food \\ Fortification with Bovine Hemoglobin (Preliminary Study)
}

Nadir N. Nogueira "; Célia Colli ${ }^{2}$ \& Silvia M. F. Cozzolino ${ }^{2}$

NOGUEIRA, N. N.; COLLI, C. \& COZZOLINO, S. M. F. Iron Deficiency Anemia Control in Pre-School Children by Food Fortification with Bovine Hemoglobin (Preliminary Study). Cad. Saúde Públ., Rio de Janeiro, 8 (4): 459-465, oct/dec, 1992.

The effect of hemoglobin-fortified cookies on hemoglobin $(\mathrm{Hb})$ levels of 16 children (2 to 4 years of age) were evaluated. The children were attending a day nursery managed under the Social Welfare Service of the State of Piaui (northeast Brazil).

All children were iron deficient according to the average transferrin saturation of $7.1+3,7 \%$, and $12(75 \%)$ of them were anemic $(\mathrm{Hb}<11 \mathrm{~g} / \mathrm{dL})$. The average $\mathrm{Hb}$ concentration was $9.4+$ $2,6 \mathrm{~g} / \mathrm{dL}$.

Cookies fortified with $3 \%$ bovine concentrate (dried by the sponted bed technique) were offered to the children over the course of 90 days (5 cookies ( $4 \mathrm{mg} \mathrm{Fe}) /$ child/d), with total iron intake of approximately $8 \mathrm{mg} \mathrm{Fe} / \mathrm{d}$.

After the experimental period the levels of $\mathrm{Hb}$ in all children had risen to levels above $11.0 \mathrm{~g} / \mathrm{dL}$ (average $13,2+0,2 \mathrm{~g} / \mathrm{dL}$ ).

The results demonstrate the possibility of using dried $\mathrm{Hb}$ concentrate in food-fortification programs aimed at meeting daily iron requirements for pre-school and school children in Brazil. Keywords: Fortification; Iron; Anemia; Pre-School Children; Bovine Hemoglobin

\section{INTRODUÇÃO}

A fortificação de alimentos com ferro é a estratégia indicada pelas instituições que atuam na área de saúde como a mais eficiente para o combate à anemia por deficiência de ferro (World Health Organization, 1989). O tema vem sendo abordado por muitos pesquisadores e já se conhece as pontencialidades de diversos sais de ferro como, agentes de ferro biodisponí-

\footnotetext{
${ }^{1}$ Departamento de Nutrição do Centro de Ciências da Saude da Universidade Federal do Piaul. Campus Universitário Iningá, Bairro Iningá, 64050, Teresina, PI, Brasil.

2 Departamento de Alimentos e Nutrição Experimental da Faculdade de Ciências Farmacêuticas da Universidade de São Paulo. Cidade Universitária "Armando de Salles Oliveira", Caixa Postal 66355, 05389-970. São Paula, $S P$, Brasil.
}

vel na fortificação de alimentos (Cook, 1973; Cook et al., 1983; Dallman et al., 1980; Fritz et al., 1970; Skikne, 1988). Uma outra forma de ferro de fortificação não-convencional é o concentrado de ferro hemoglobínico, obtido do sangue de abate de animais. Os principais trabalhos nessa área foram desenvolvidos no Chile, onde foi avaliada a biodisponibilidade de ferro hemínico em alguns alimentos (leite, biscoito, cereal extrusado) (Asenjo et al., 1985; Calvo et al., 1989; Hertrampf, 1982; Hertrampf et al., 1990).

Como subproduto do abate de animais, o sangue é uma excelente fonte de ferro hemínico e de proteínas para o enriquecimento de alimentos (Domene, 1988; Piske, 1982). O volume coletado por animal representa aproximadamente 3,5\% de seu peso vivo. No Brasil, em 1984, foi estimado que cerca de 130 milhões de litros 
de sangue são quase que totalmente descartados na maioria dos matadouros do país; esse sangue de abate é um poluente em potencial dos mananciais e também responsável pelo alto custo do tratamento dos efluentes dos abatedouros (Ré, 1986). Por sua vez, somente uma pequena quantidade desse volume é utilizada para consumo humano, fato decorrente das dificuldades tecnológicas para a secagem do sangue e das alterações organolépticas decorrentes de sua adição ao alimento. A técnica de secagem em leito de jorro, (Ré, 1986) mostrou-se uma alternativa de menor custo em relação à secagem em spray, fornecendo um produto adequado para o consumo humano.

Propostas de desenvolver, em nosso país, alimentos fortificados que possam ser introduzidos em programas de combate à anemia por deficiência de ferro atendem a uma necessidade evidenciada pelos estudos de prevalência realizados, que, embora restritos a algumas regiões, assinalam prevalências da ordem de $30 \% \mathrm{em}$ crianças de até 6 anos de idade (Cook, 1977; Gandra et al., 1977; Salzano et al., 1985; Sigulem et al., 1978). Propusemo-nos, assim, a avaliar o efeito da introdução de biscoito fortificado com concentrado de hemoglobina bovina no estado de nutrição de ferro de pré-escolares, - num estudo preliminar que serviria de indicação para trabalhos futuros nesta área.

\section{MATERIAL E MÉTODOS}

A amostra inicial foi de 30 crianças, de ambos os sexos, na faixa etária de 2 a 4 anos incompletos, atendidas numa creche no estado do Piauí.

No decorrer do trabalho, 14 das crianças estudadas sairam da creche por terem atingido a idade máxima permitida pelo serviço de atendimento, ficando, portanto, a amostra reduzida a 16 crianças.

Essas crianças, filhas de mães operárias, permanecem na creche por um periodo de 10 horas diárias (7:30 - 17:30 horas), de segunda a sexta-feira, e no local são oferecidas cinco refeições diárias.

As mães ou responsáveis pelas crianças foram esclarecidas sobre o objetivo da pesquisa, assinando um termo de ciêtı ‘a e concordância com o estudo. Antes do início da intervenção, foi feita uma pesquisa parasitológica de fezes (Minami, 1983) em amostras coletadas por três dias consecutivos, não sendo detectada a presença de parasitas relacionados à etiologia da anemia. Após esse período, procedeu-se a uma coleta de sangue (pré-intervenção) para determinação dos parâmetros hematológicos ligados ao estado de nutrição de ferro. As crianças passaram a consumir, diariamente, além da dieta normal, 5 (cinco) biscoitos fortificados que aportavam, aproximadamente, $4 \mathrm{mg}$ de ferro. Após 90 dias, procedeu-se a uma nova coleta de sangue (pós-intervenção).

Foram coletados $10 \mathrm{ml}$ de sangue venoso das crianças, em jejum de, no mínimo, 10 horas. Cerca de $1 / 3$ desse volume foi colocado em frasco com anticoagulante (EDTA 8\%) para determinações de hemoglobina e hematócrito. $O$ volume restante foi transferido para tubos desmineralizados, sendo separado o soro após centrifugação.

A concentração de hemoglobina e o volume hematócrito foram determinados pelos métodos da ciano-meta-hemoglobina e macro-hematócrito, respectivamente (Miali, 1977).

A concentração de ferro sérico e a capacidade total de fixação de ferro foram determinadas por métodos propostos pelo International Committee for Standardization in Haematology (1978a, 1978b).

\section{OBTENÇÃO DOS BISCOITOS}

\section{Coleta e Secagem do Sangue Bovino}

A coleta de sangue foi realizada em um abatedouro de animais, sob inspeção de um médico veterinário responsável pelo setor. Utilizou-se como anticoagulante uma mistura de citrato de sódio, cloreto de sódio e dextrose $(0.8: 1,5: 4,0)$, colocada no sangue na proporção de 1:4. Para evitar o crescimento microbiano, foi adicionado sulfato de estreptomicina à concentração de $0,1 \%$, a qual foi, em seguida armazenada a $4^{\circ} \mathrm{C}$. Posteriormente, procedeu-se à secagem pelo processo de leito de jorro, em equipamento adaptado para a secagem de sangue por Ré (1986). O produto assim obtido, contendo, segundo esses autores, $87 \%$ de proteí- 
nas e $0,22 \%$ de ferro, mostrou-se aceitável de acordo com os padrões microbiológicos oficiais estabelecidos para a suplementação de alimentos.

\section{Preparação dos Biscoitos}

Os biscoitos foram preparados em uma indústria de alimentos seguindo a formulação da Tabela 1. Utilizou-se as temperaturas de $100^{\circ} \mathrm{C}$ à entrada do forno, $200^{\circ} \mathrm{C}$ na porção intermediária, e $100^{\circ} \mathrm{C}$ à saída.

Utilizando-se amostras aleatorizadas, a concentração de ferro dos biscoitos foi determinada por espectrofotometria de absorção atômica (espectrofotômetro Perkin-Helmer mod. 373), após digestão por via úmida, com $\mathrm{HNO}_{3} \mathrm{e}$ $\mathrm{HClO}_{4}(5: 1)$.

TABELA 1. Formulação de Biscoitos Fortificados com Ferro Hemínico

\begin{tabular}{lr}
\hline \hline Item & $\%$ \\
\hline Farinha de Trigo & 70,00 \\
Gordura hidrogenada & 1,50 \\
Açúcar & 26,00 \\
Lecitina & 0,50 \\
Bicabornato de amônio & 0,70 \\
Bicabornato de sódio & 0,25 \\
Bissulfito de sódio & 0,04 \\
Benzoato de sódio & 0,02 \\
Cloreto de sódio & 0,50 \\
BHT/BHA & 0,01 \\
Sangue bovino seco & 3,00 \\
\hline \hline
\end{tabular}

* (1:1)

TABELA 2. Concentração de Hemoglobina (Hb), Hematócrito (Htc), Ferro Sérico (FeS) Capacidade

Total de Fixação de Ferro (CTFF), Saturação de Transferrina (ST) das Crianças de 2 a 4 anos de Idade Atendidas pela Creche (antes da intervenção).

\begin{tabular}{lccccc}
\hline \hline & HB & Htc & FeS & CTFF & ST \\
& $\mathrm{g} / \mathrm{dL}$ & $\%$ & $\mathrm{ug} / \mathrm{dL}$ & $\mathrm{ug} / \mathrm{dL}$ & $\%$ \\
\hline $\bar{X}+\mathrm{S}$ & $9,4+2,6$ & $31+2$ & $23+13$ & $326+25$ & $7,1+3,7$ \\
Intervalo & $(9,0-11,3)$ & $(28-35)$ & $(12-47)$ & $(285-388)$ & $(3,8-14,1)$ \\
$\mathrm{n}$ & 16 & 16 & 11 & 11 & 11 \\
Valores Limites & 11 & 33 & 50 & 400 & 15 \\
\hline \hline
\end{tabular}

\section{RESULTADOS E DISCUSSÃO}

Usando a concentração de hemoglobina $(\mathrm{Hb})$ como único parâmetro para o diagnóstico de anemia nas crianças estudadas, encontramos 12 delas (75\%) anêmicas $(\mathrm{Hb}<11 \mathrm{~g} / \mathrm{dL})(20)$. A média encontrada foi de $9,4+2,6 \mathrm{~g} / \mathrm{dL}$, com intervalo entre $9 \mathrm{~g} / \mathrm{dL}$ e $11,3 \mathrm{~g} / \mathrm{dL}$. Os outros parâmetros hematológicos confirmaram um estado de depleção de ferro, caracterizando um quadro de anemia por deficiência deste mineral (Tabela 2).

A avaliação das dietas fornecidas na creche, durante uma semana (Tabela 3), mostra uma notória monotonia, sobretudo nas refeiçōes principais. A quantidade de verduras e de hortaliças consumidas é baixa e praticamente não há a presença de frutas nas dietas, o que compromete o aporte de vitaminas e de minerais. Alimentos de origem animal (leite e carnes) aparecem na dieta, diariamente, em pequena quantidade. Os resultados da análise química dessa dieta, comparados com as recomendações de nutrientes do National Research Council (1989) (Tabela 4), mostram um importante déficit no consumo calórico e na adequação protéica, o qual certamente tem influência no estado geral de saúde destas crianças. $O$ fato de haver adequação protéica não significa que o organismo a utilize completamente com finalidade plástica, pois certamente uma parte estará sendo comprometida com o fornecimento de energia. Quadro semelhante foi encontrado em dietas de pré-escolares de outras regiões do país com condições de atendimento semelhantes (Hertrampf et al., 1990; Mazzili, 1987; Salzano et al., 1985; Szarfarc et al., 1988). 
O resultado da análise química da dieta mostrou uma inadequação de ferro da ordem de $60 \%$ (4 mg Fe/d) confirmando resultado de outros autores (Mazzili, 1987; Sichieri, 1987) para esse grupo etário e justificando a elevada proporção de anemia encontrada. Em condições normais, o pré-escolar raramente tem atendidas suas necessidades de ferro exclusivamente com a dieta, particularmente quando esta é quantitativamente insuficiente. Trabalho realizado por Szarfarc et al. (1988) demonstrou que apenas $13,7 \%$ das dietas infantis avaliadas no municipio de São Paulo satisfaziam a recomendação (10 mg Fe/d).

TABELA 3. Dieta Fornecida na Creche

\begin{tabular}{lll}
\hline \hline Refeiçóes & Preparações & $\begin{array}{l}\text { Quantidade } \\
\text { Per capita (g) }\end{array}$ \\
\hline Desjejum & $\begin{array}{l}\text { Mingau de maisena } \\
\text { ou } \\
\text { leite }\end{array}$ & 137 \\
& e & 100 \\
& Beiju de tapioca & 31 \\
Lanche & $\begin{array}{l}\text { K-suco de fruta } \\
\text { ou }\end{array}$ & 127 \\
& $\begin{array}{l}\text { polenta } \\
\text { Almoço }\end{array}$ & $\begin{array}{l}\text { Arroz com feijão } \\
\text { carne bovina }\end{array}$ \\
& $\begin{array}{l}\text { Sopa de merenda } \\
\text { Jantar }\end{array}$ & 193 \\
& $\begin{array}{l}\text { escolar suplementada } \\
\text { com verduras e carne }\end{array}$ \\
\hline \hline
\end{tabular}

TABELA 4. Compasição da Dieta Oferecida na Creche

\begin{tabular}{lcc}
\hline \hline \multicolumn{2}{l}{ Composição da Dieta } & Total Diário \\
\hline Energia (b) & $(\mathrm{kcal})$ & 540,0 \\
Proteina (c) & $(\mathrm{g})$ & 23,0 \\
Lipidio & $(\mathrm{g})$ & 16,5 \\
Carboidrato & $(\mathrm{g})$ & 75,0 \\
Fibra & $\ldots . .(\mathrm{g})$ & 19,0 \\
Minerais & $(\mathrm{g})$ & 13,0 \\
\hline Ferro (d) & $(\mathrm{mg})$ & 4,0 \\
\hline \hline
\end{tabular}

(a) Para uma ingestão média diária de $760 \mathrm{~g}$

(b) (c) (d) Ingestão diária recomendada (RDA1989) $1300 \mathrm{Kcal} 16 \mathrm{~g}$ proteína, $10 \mathrm{mg} \mathrm{Fe}$.

(National Research Council, 1989)
Através da análise química do biscoito fortificado, verifica-se que seu acréscimo na dieta da creche elevou a adequação de ferro para $83 \%$ $(8,3 \mathrm{mg} / \mathrm{d})$ (Tabelas 5 e 6$)$. Ao se comparar a alimentação institucional com e sem biscoito fortificado, utilizando o modelo proposto por Monsen (1978) para a avaliação do ferro absorvível (Tabela 6), verifica-se que este passa de $0,3 \mathrm{mg}$ para $1,2 \mathrm{mg}$, valor que atende à demanda da criança pelo mineral $(1,0 \mathrm{mg})$.

TABELA 5. Composição Química do Biscoito Fortificado com 3\% de Concentrado de Hemoglobina ( $\mathrm{CHb}$ )

\begin{tabular}{lr}
\hline \hline Composição* & Quantidades \\
\hline Energia (Kcal) & 127,0 \\
Proteína (g) & 5,0 \\
Lipídio (g) & 1,0 \\
Carboidrato (g) & 24,5 \\
Cinza (g) & 0,3 \\
Umidade (g) & 4,0 \\
\hline Ferro (mg) & 4,2 \\
\hline \hline
\end{tabular}

* Para uma ingestão média diária de $35 \mathrm{~g}$ de biscoito

TABELA 6. Indicadores de Ferro na Dieta sem e com Adição do Biscoito Fortificado

\begin{tabular}{lcc}
\hline \hline Indicadores & $\begin{array}{c}\text { Dieta s/ } \\
\text { Biscoito }\end{array}$ & $\begin{array}{l}\text { Dieta c/ } \\
\text { Biscoito }\end{array}$ \\
\cline { 2 - 3 } & $\mathrm{X}$ & $\mathrm{X}$ \\
\hline Ferro total (mg) & 4,0 & $\mathbf{8 , 3}(\mathrm{a})$ \\
Ferro absorvivel (b) & 0,3 & 1,2 \\
\hline \hline
\end{tabular}

(a) Recomendação $10 \mathrm{mg} \mathrm{Fe} / \mathrm{d}$ (National Research Council, 1989)

(b) Cálculo segundo o modelo sugerido por Monsen, et al. (1978)

Os resultados observados mostram que a população submetida à intervenção respondeu com rapidez à oferta de ferro, sendo que, no periodo de 3 (três) meses, houve uma recuperação dos niveis de hemoglobina em todas as crianças (Figura 1). Embora tenha-se conhecimento de que a frequiência de anemia diminui nesta faixa etária, podendo levar à suposição que o efeito observado poderia ser normal 
FIGURA 1. Concentração de Hemoglobina (Hb) e Hematócrito (Htc) de Crianças (2 a 4 anos), antes e após a Intervenção (5 biscoitos/d, 3 meses, $n=16$ ).
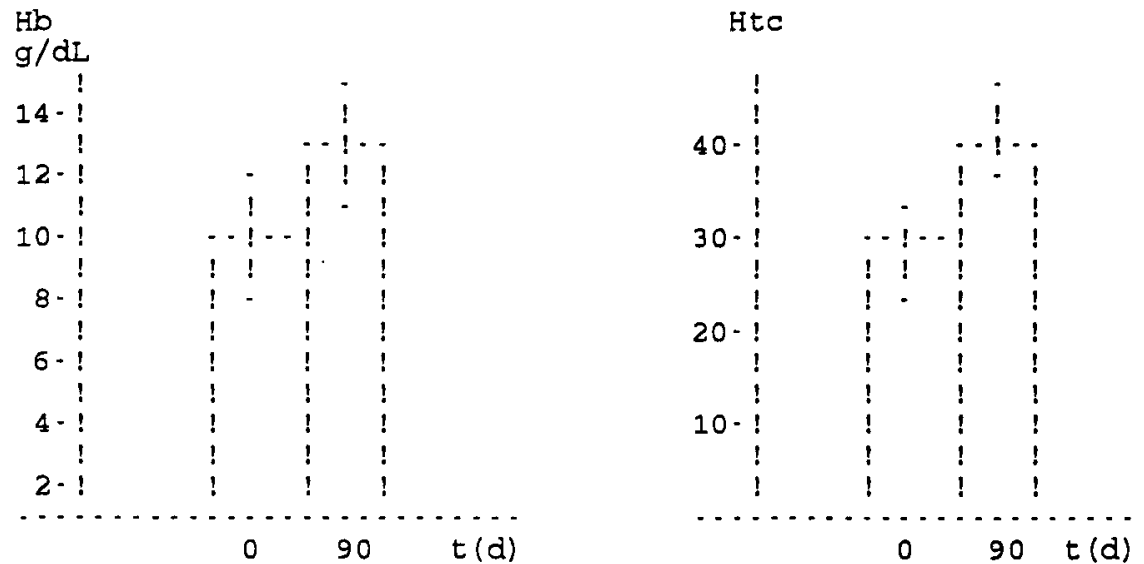

Observaçōes: A concentração de hemoglobina é um dos ûltimos parametros a ser alterado na deficiéncia de ferro e o primeiro a ser observado na recuperação. Neste estudo, procuramos observar a biodisponibilidade do ferro hemínico na recuperação da anemia, utilizando apenas a hemoglobina como parametro. Outros trabalhos estão sendo conduzidos em nosso laboratório para verificar o efeito da adiçåo de ferro hemínico a outros alimentos e também os depósitos deste elemento no organismo.

mesmo sem a suplementação, este aspecto não deve ser considerado, uma vez que o período de tempo do estudo foi curto e o resultado foi positivo em $100 \%$ das crianças anêmicas.

É importante referir que o trabalho de esclarecimento feito com as mães foi fundamental para assegurar o consumo diário durante o período de intervenção, inclusive nos fins de semana. Sem este, a menção de se utilizar biscoitos fortificados com sangue bovino poderia esbarrar em tabus, modificando a adesão de $100 \%$ encontrada para o consumo do produto fortificado. A partir dos resultados obtidos, pretende-se avaliar a eficiência desse tipo de intervenção em grupos maiores.

A possibilidade de se utilizar, no Brasil, o subproduto da indústria de abate de animais como fonte de ferro na fortificação de alimentos vem ao encontro da necessidade de combate à anemia ferropriva em nossa população. Outros possiveis veículos alimentares devem ser avaliados para aumentar o leque de alternativas que objetivam o controle dessa patologia.

\section{AGRADECIMENTOS}

Ao Dr. Alberto Tavares Silva, Governador do Estado do Piauí, ao Serviço Social do Estado do Piauí, ao Prof. Carlos Alberto Bragança do Instituto de Matemática e Estatística da Universidade de São Paulo, aos Laboratórios de Bioquímica Clínica e Hematologia Clínica da Faculdade de Ciências Farmacêuticas da Universidade de São Paulo e do Estado do Piauf pelo auxilio na execução do trabalho, a Maria da Conceição Pereira Nunes e Isabel Cristina Bossi Alves pelo trabalho de secretaria.

\section{RESUMO}

NOGUEIRA, N. N.; COLLI, C. \& COZZOLINO, S. M. F. Controle da Anemia Ferropriva em Pré-Escolares por Meio da Fortificação de Alimento Com Concentrado de Hemoglobina Bovina (Estudo

Preliminar). Cad. Saúde Públ., Rio de Janeiro, 8 (4): 459-465, out/dez, 1992.

$O$ efeito da introdução de biscoitos fortificados com ferro hemínico no estado de nutrição de ferro de pré-escolares foi avaliado em um estudo piloto que inclui 16 crianças, com idade entre 2 e 4 anos, de uma creche pública do estado do Piauí. A fonte de ferro utilizada foi o sangue bovino seco pelo processo de leito de jorro, uma alternativa 
para a secagem em spray, adaptada para a secagem de sangue. À primeira tomada de amostra, detectou-se anemia $(\mathrm{Hb}<11 \mathrm{~g} / \mathrm{dL})$ em 12 crianças (75\%). $\mathrm{O}$ valor médio de $\mathrm{Hb}$ foi de $9,4 \quad 2,6 \mathrm{~g} / \mathrm{dL}$. Os biscoitos fortificados com $3 \%$ de concentrado de hemoglobina bovina foram introduzidos na dieta oferecida ( 5 biscoitos (4mg Fe)/d) durante 3 meses. Após esse periodo, houve aumento da concentração de hemoglobina em todas as crianças e ausência de anemia $(\mathrm{Hb}=$ $13,20,2 \mathrm{~g} / \mathrm{dL}$ ). Os resultados obtidos apontam para a utilização do sangue total seco como uma fonte de ferro hemínico possível de ser utilizada na fortificação de alimentos, principalmente dirigidos a grupos de risco de anemia por deficiência de ferro, como é o caso dos pré-escolares.

Palavras-Chave: Fortificação; Ferro; Anemia; Pré-Escolares; Hemoglobina Bovina

\section{REFERÊNCIAS BIBLIOGRÁFICAS}

ASENJO, J. A.; AMAR, M.; CARTAGENA, N.; KING, J.; HICHE, E. \& STEKEL, A., 1985. Use of a bovine heme iron concentrate in the fortification of biscuits. Journal of Food Science, 50: 795-799.

CALVO, E.; HERTRAMPF, E.; PABLO, S.; AMAR, M. \& STEKEL, A., 1989. Haemoglobin fortified cereal: an alternative weaning food with high iron bioavailability. European Journal of Clinical Nutrition, 43: 237-243.

COOK, J. D.; MINNICH, V.; MOORE, C. V.; RASMUSSEN, A.; BRADLEY, W.B. \& FINCH, C. A., 1973. Absorption of fortification iron in bread. American Journal of Clinical Nutrition, 26: 861-872.

COOK, J. D., 1977. Absorption of food iron. Federation Proceedings, 36: 2028-2032.

COOK, J. D. \& REUSSER, M. E., 1983. Iron fortification: an update. American Journal of Clinical Nutrition, 38: 648-659.

DALLMAN, P. R.; SIIMES, M. A. \& STEKEL, A., 1980. Iron deficiency in infancy and childhood. American Journal of Clinical Nutrition, 33: 86-118.

DOMENE, S. M. A., 1988. Utilização de sangue bovino para alimentação humana. Revista de $\mathrm{Nu}$ trição da Pontificia Universidade Católica de Campinas (Puccamp), 1: 163-179.
FRITZ, J. C.; PLA, G. W.; ROBERTS, T.; HOUE, E. L. \& BOEHNE, J. W., 1970. Biological availability in animals of iron from common dietary sources. Journal of Agricultural and Food Chemistry, 18: 647-651.

GANDRA, Y. R. \& SALZANO, A. C., 1977. Seminário sobre anemias nutricionais no Brasil: relatório final. Braślia: Instituto Nacional de Alimentação e Nutrição.

HERTRAMPF, E., 1982. Utilización de Sangre Bovina en la Fortificación de la Leche. Tese de Mestrado, Santiago: Instituto de Nutrição e Tecnologia de Alimentos.

HERTRAMPF, E.; OLIVARES, N.; PIZARRO, F.; WALTER, T.; CAYAZZO, M.; HERESI, G.; LLAGUNO, S.; CHADUD, P. \& STEKEL, A., 1990. Hemoglobin fortified cereal: A source of available iron to breast-fed infants. European Journal of Clinical Nutrition, 44: 793-798.

INTERNATIONAL COMMITTEE FOR STANDARDIZATION IN HAEMATOLOGY, 1978a. The measurement of total and unsaturated iron-binding capacity in serum. British Journal of Haematology, 38: 281-290.

, 1978b. Recommendations for measurement of serum iron in human blood. British Journal of Haematology, 38: 291-294.

MAZZILI, R. N., 1987. Valor nutricional da merenda escolar e sua contribuição para as recomendações nutricionais do pré- escolar matriculado em CEAP. Revista de Saúde Pública, 21: 246-254.

MIALI, J. B., 1977. Laboratory Medicine Haematology. 5! ed., Saint Louis: Mosby.

MINAMI, P. S., 1983. Coletanea de Técnicas Parasitológicas. São Paulo: Faculdade de Ciências Farmacêuticas.

MONSEN, E. R.; HALLBERG, L.; LAYRISSE, M.; HEGSTED, D. M.; COOK, J. D.; MERTZ, W. \& FINCH, C. A., 1978. Estimation of available dietary iron. American Journal of Clinical Nutrition, 41: 134-141.

MONTEIRO, C. A. \& SZARFARC, S. C., 1987. Estudo das condições de saúde das crianças do município de São Paulo. V - Anemia. Revista de Saúde Pública, 21: 255-260.

NATIONAL RESEARCH COUNCIL, 1989. Recommended Dietary Allowances. 10" ed., Washington: National Academy Press.

ORGANIZACIÓN MUNDIAL DE LA SALUD, 1975. Lucha contra la anemia nutricional, especialmente contra la carencia de hierro. Geneve, 71p. Informe de una reunión mixta ADI/OEA/ OMS. (Série de Informes Técnicos, 580). 
PISKE, D., 1982. Aproveitamento de sangue de abate para alimentação humana. I - Uma revisão. Boletim do Instituto de Tecnologia de Alimentos, 19: 253-308.

RÉ, M. I., 1986. Secagem de Sangue Animal em Leito de Jorros. Tese de Mestrado, São Carlos: Centro de Ciências e Tecnologia, Universidade Federal de Såo Carlos.

SALZANO, A. C.; TORRES, M. A. A.; BATISTA FILHO, M. \& ROMANI, S. A. M., 1985. Anemias em crianças de dois serviços de saúde de Recife, PE (Brasil). Revista de Saúde Pública, 19: 499-507.

SICHIERI, R., 1987. Anemia Nutricional em Crianças Menores de 5 Anos do Municipio de Säo Paulo: papel da dieta na determinação de sua prevaléncia. Tese de Doutorado, São Paulo: Faculdade de Saúde Pública, Universidade de Såo Paulo.
SIGULEM, D. M.; TUDISCO, E. S.; GOLDENBERG, P.; ATHAIDE, M. M. M. \& VAISMAN, E., 1978. Anemia fertopriva em crianças do município de São Paulo. Revista de Saude Pública, 12: 168-178.

SKIKNE, B. S., 1988. Current concepts in iron deficiency anemia. Food Review International, 4: 137-173.

SZARFARC, S. C.; MONTEIRO, C. A.; MEYER, M.; TUDISCO, E. S. \& REIS, I. M., 1988. Estudo das condiçóes de saúde das crianças do município de Såo Paulo, SP (Brasil), 1984/1985. X - Consumo Alimentar. Revista de Saúde Pública, 22: 266-272.

WORLD HEALTH ORGANIZATION, 1989. Preventing and Controlling Iron Deficiency Anaemia Through Primary Health Care. A guide for health administrators and programme managers. Geneve: WHO. 